LAWRENCE LIVERMORE N A TIO N A L LABORATORY

\section{ICF Program: LDRD-ER Final Report}

S. H. Glenzer

February 20, 2004 
This document was prepared as an account of work sponsored by an agency of the United States Government. Neither the United States Government nor the University of California nor any of their employees, makes any warranty, express or implied, or assumes any legal liability or responsibility for the accuracy, completeness, or usefulness of any information, apparatus, product, or process disclosed, or represents that its use would not infringe privately owned rights. Reference herein to any specific commercial product, process, or service by trade name, trademark, manufacturer, or otherwise, does not necessarily constitute or imply its endorsement, recommendation, or favoring by the United States Government or the University of California. The views and opinions of authors expressed herein do not necessarily state or reflect those of the United States Government or the University of California, and shall not be used for advertising or product endorsement purposes.

This work was performed under the auspices of the U.S. Department of Energy by University of California, Lawrence Livermore National Laboratory under Contract W-7405-Eng-48. 


\section{ICF Program: LDRD-ER Final Report}

Title: Non-linear saturation of parametric laser-plasma instabilities

Tracking No. (if continuing): $\quad$ 01-ERD-107

Principle Investigator:

Division:

Phone:

Mail Station:

Co-investigators:
Siegfried H. Glenzer

ICF/NIF

2-7409

L-399

R. L. Berger (D\&NT)

L. M. Divol (PAT)

D. H. Froula (UC Davis student)

\section{Summary}

In the 01-ERD-107 LDRD-ER project, we have performed novel Thomson scattering experiments at the Trident and Omega laser facilities and provided high quality spectral data. These results have led to the development of the first quantitative understanding of laser-plasma interactions for NIF plasmas. For this purpose an green/ultraviolet probe laser, built for Nova in 1998 [1] and successfully used to measure both temperature and plasma wave amplitudes [2], has been deployed on Omega. The Thomson scattering diagnostics has been used twofold: 1) it provided independent measurements of the plasma electron and ion temperature, the plasma flow velocity, or the electron distribution function; 2) it provided measurements of the primary plasma wave and their secondary non-linear decay wave products. These experiments at Omega provide definitive quantitative results on the nonlinear saturation of stimulated Raman scattering for green $(2 \omega)$ beams [3]. In addition, the experiments on the Trident laser have led to a quantitative understanding of the stimulated Brillouin scattering [4-7] in low-Z plasmas. A nonlinear frequency detuning model has successfully explained all the experimental observable including the SBS reflectivity. This model has been implemented into the laser-plasma interaction code $\mathrm{pF} 3 \mathrm{D}$ as a tool to design and optimize NIF target experiments with SBS and SRS losses included. The development of quantitative models for SBS and SRS for various regimes has now been adopted as part of the WBS1 project within the ICF program.

\section{Introduction}

The properties of plasma waves that nonlinearly scatter the laser light in plasma conditions are of general interest to the inertial confinement fusion, high energy density, and nuclear weapons effects (ICF, HED, and NWET) communities. The instabilities of primary concern in the field of laser-plasma interactions are stimulated Raman and 
Brillouin scattering (SRS and SBS). For ICF, these processes can reflect a large fraction of the incident laser light thereby reducing the efficiency of the target, affecting implosion symmetry, inducing laser damage and causing capsule pre-heat. For HED, large scatter will reduce the achievable radiation temperature and possibly produce non-

optimum X-radiation spectra. However, for NWET, large SRS may produce interesting $\mathrm{keV} \mathrm{X}$-ray fluxes. In this work, both the spatial and temporal evolution of the plasma waves has been measured and correlated with the time resolved SBS and SRS. Important for these studies are the behavior of the plasma waves as the electron temperature, plasma density, material, and laser intensity varies. For laser intensities of interest for higher temperature ignition hohlraums of $300 \mathrm{eV}$ to $350 \mathrm{eV}$ we found that these wave amplitudes are saturated. As of yet unobserved plasma wave decay products and modification of the distribution functions that are conjectured to be responsible for saturation of parametric laser-plasma interaction instabilities have indeed been observed in this LDRD project [8]. Measurements of the density fluctuations in the plasma, available with Thomson scatter, were required to discover the physics governing the saturation processes. Such Thomson scattering measurements were begun on Nova where a prototype design of the system we have implemented for Omega and Trident was successfully used on multiple experiments. In support of this experimental initiative, $\mathrm{pF} 3 \mathrm{D}$ simulations [9] of the existing data base of SRS and SBS from gasbags, have been performed with realistic models of the laser beam and with saturation models developed in this period $[4,7,8,10]$. These models are continuously being improved based on calculations by our collaborators at Princeton [11], Ecole Polytechnique [12], LANL [13], LLE, University of Alberta, and UCLA.

\section{Progress of Modeling Nonlinear Interactions}

It is now well established that the stimulated Raman and Brillouin scattering instabilities will be saturated in the high temperature plasma regime produced on the next generation of large laser facilities for ICF. In these instabilities, ion acoustic waves or electron plasma waves are excited to large amplitudes by the incident light wave that then cause the reflection of the incident wave. The experimental evidence for saturation consists of reflectivity measurements that show no growth with increasing laser intensity and in several cases of Thomson scattering measurements of the primary ion-acoustic wave amplitude as well as of the primary electron plasma wave. Simulations and theoretical modeling indicate that saturation in low- $Z$ or in high- $Z$ plasmas is caused by different processes. In low-Z plasmas, trapping of ions in the ion-acoustic wave flattens the ion velocity distribution function at the ion sound speed inducing a frequency and $\mathbf{k}$-vector mismatch for the parametric SBS instability. Our model of this de-tuning process as implemented into the laser-plasma interaction code $\mathrm{pF} 3 \mathrm{D}$ is indicated in Fig. 1. With increasing wave amplitude the frequency of the wave is calculated to vary by up to $30 \%$ which is sufficient to saturate SBS. Moreover, recent PIC simulations have indicated that a frequency mismatch is followed by the decay of large amplitude ion acoustic waves into two daughter ion waves [10]. This non-linear saturation mechanism directly limits the amplitude of the ion acoustic waves. In recent experiments performed in this LDRD we have indeed observed the two-ion decay instability. This is an experimental milestone for the development of quantitative saturation models in high- $Z$ plasmas. 
Similarly, it has also been proposed that the sideband instability directly limits the amplitude of electron plasma waves [11]. This saturation process primarily applicable for high-Z plasma has also been implemented into our modeling (cf. Fig. 1) where the damping of the primary wave is calculated to rise rapidly at the threshold for the onset of the decay process. However, up to now, there has been no direct measurements of the side band instability associated with saturation in high-temperature fusion plasmas and consequently, there are no quantitative tests available to establish the coefficients used in this particular saturation model.
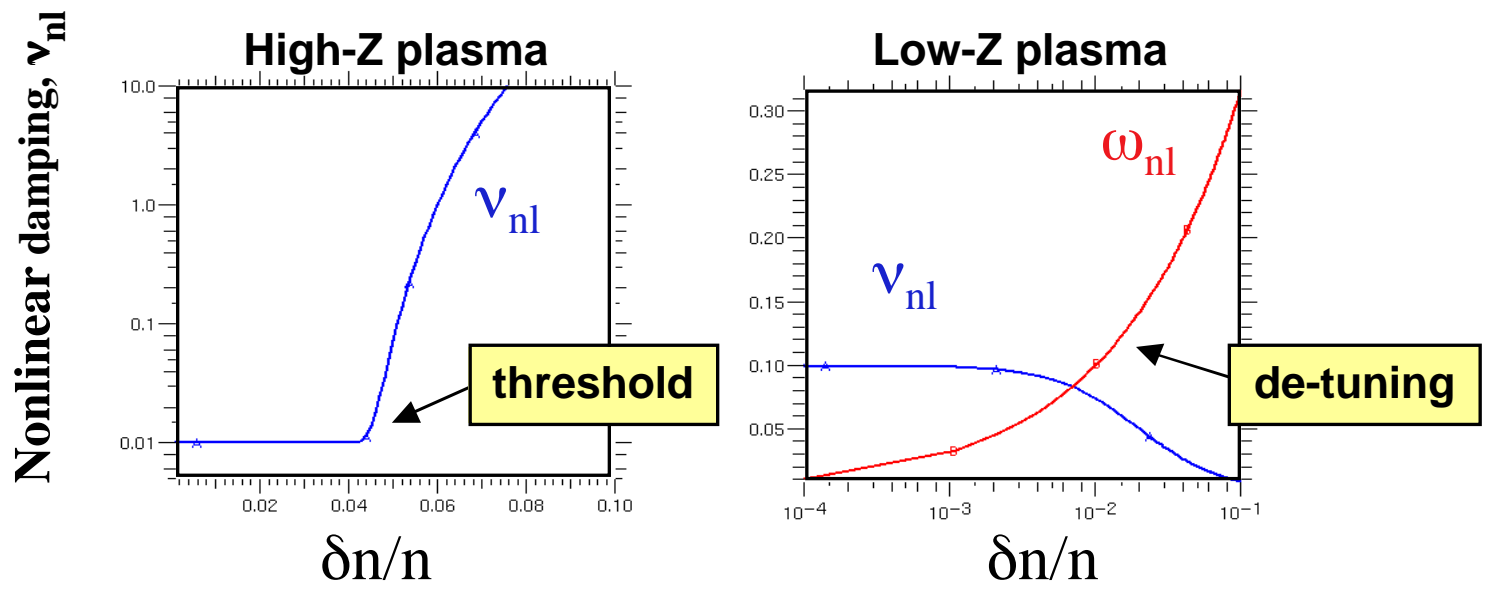

Figure 1. Nonlinear saturation physics modeled in our parallel laser-plasma interaction code $\mathrm{pF} 3 \mathrm{D}$. The nonlinear damping, $v_{\mathrm{nl}}$, and frequency of the plasma waves, $\omega_{\mathrm{n} l}$, is included in the coupled wave equations describing the interaction of laser light with the plasma. In high-Z plasmas, the nonlinear damping, $v_{n}$, is strongly increased when the wave amplitudes reaches the threshold while in low-Z plasmas a spatial and temporal de-tuning occurs saturating the laser backscatter.

On the other hand, the saturation model for SBS in low-Z plasmas is now well established, tested and included in $\mathrm{pF} 3 \mathrm{D}$ with coefficients that provide reliably quantitative agreement with experiments [14]. This so-called dynamic ion trapping model reproduces the modifications of the background ion distribution function seen in PIC simulations (see Fig. 2) and their effect on the dispersion relation for SBS-driven acoustic waves. It consists in an evolution equation for the width of the velocity-plateau created by trapping around the phase velocity of the acoustic wave. The resulting frequency shift and modification of the Landau damping are then included in the propagation equation for the SBS-driven acoustic wave. Parallelization has been straightforward. The tests have been successfully completed. Numerical stability when the nonlinear frequency shift induced by trapping becomes large has been avoided by adding a clamp on acoustic waves at large amplitude $(\delta \mathrm{n} / \mathrm{n} \sim 0.5)$ which is justified by wave-breaking, for example. 


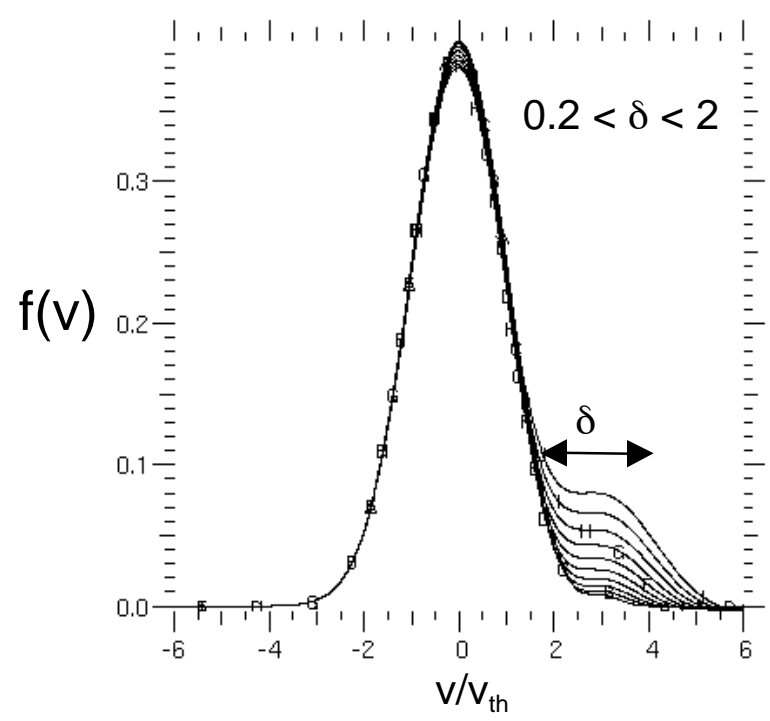

Figure 2. Our reduced model follows the evolution of the velocity plateau $\delta$ and assesses its impact on the dispersion of acoustic waves (i.e. frequency shift).

The model has been benchmarked against PIC simulations. Comparisons are being made with Bzohar 1D PIC simulations. This is not straightforward as it is difficult to reproduce the Bzohar acoustic noise source in $\mathrm{pF} 3 \mathrm{D}$. This was accomplished by fitting the noise level in $\mathrm{pF} 3 \mathrm{D}$ using the Thomson scattering limit. The intensity was then increased to drive SBS. At low intensity $\left(1.2510^{14} \mathrm{~W} . \mathrm{cm}^{-2}\right)$ and for Trident-like conditions, the agreement between the reduced model and Bzohar was quantitatively good. We further pursued this by varying the plasma parameters and the laser intensity and found acceptable agreement with PIC results. Also, we have to restrain ourselves to parameters where the trapping induced detuning is the dominant effect in the PIC simulations as they include many other nonlinear effects that are not modeled in pF3D. Finally, the noise level is Bzohar is 3 orders of magnitude higher than the usual theoretical thermal noise used in $\mathrm{pF} 3 \mathrm{D}$, which seems to increase the effect of detuning on SBS, as one is limited to modest gain. We observe that our model provides quantitative agreement with the PIC simulations. Figure 3 shows the calculated frequency shift induced by trapping as observed in a PIC-Bzohar simulation (thick line) and as calculated by the reduced model in $\mathrm{pF} 3 \mathrm{~d}$ (thin line). The right hand side shows the resulting transmission and the SBS reflectivity as function of time. The parameters were Trident-like $\left(1.2510^{14} \mathrm{~W} \mathrm{~cm}^{-2}\right.$ of $2 \omega$ light in a Be plasma at $\mathrm{N}_{\mathrm{e}} / \mathrm{N}_{\mathrm{c}}=0.025$ with $\mathrm{T}_{\mathrm{e}}=500 \mathrm{eV}, \mathrm{T}_{\mathrm{i}}=300 \mathrm{eV}$ ). Good agreement is apparent in this figure. 

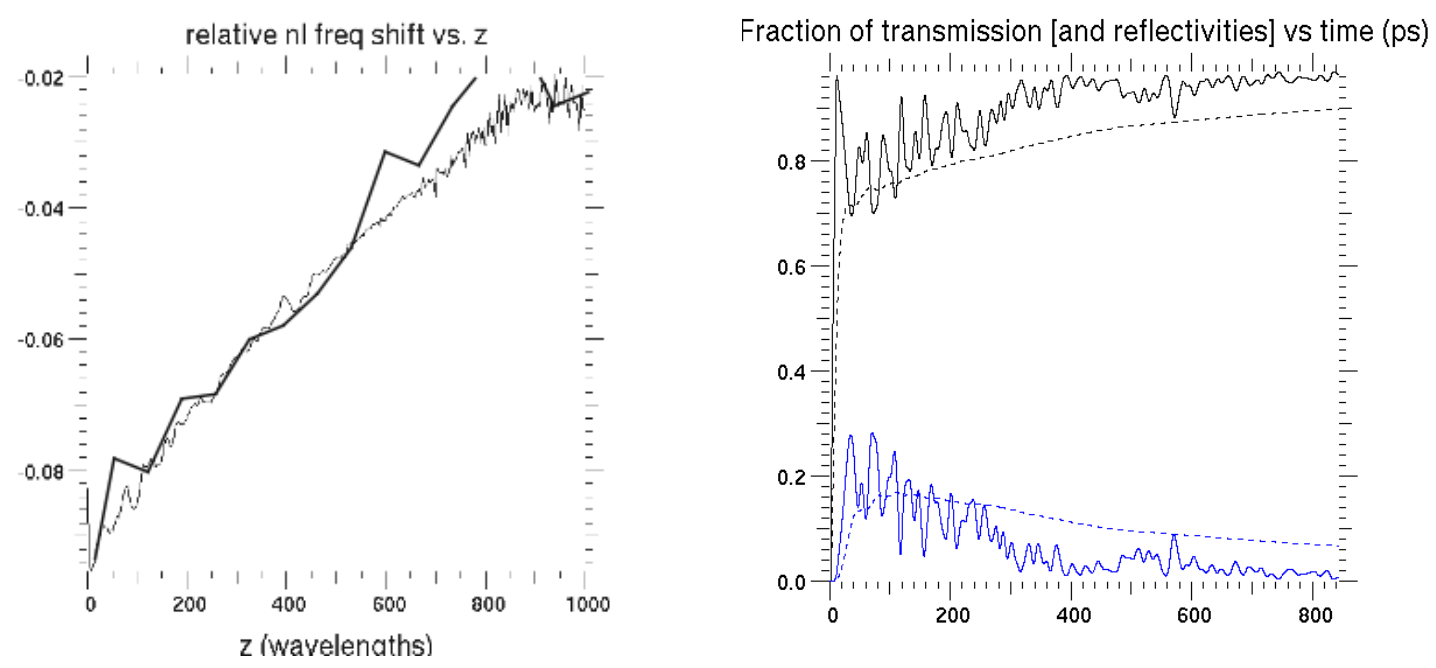

Figure 3. Left, one can compare the frequency shift induced by trapping as observed in a PIC-Bzohar simulation (thick line) and as calculated by the reduced model in $\mathrm{pF} 3 \mathrm{~d}$ (thin line). Right shows the resulting transmission and SBS reflectivity function of time.

\section{Experimental Validation of the Numerical Modeling}

The detailed predictions of the numerical model and in particular of the nonlinear frequency detuning and saturation process have been tested with experiments performed during the LDRD period. For these studies, the energy transfer in multiple and single species plasmas has been compared with simulations that include the ion wave frequency detuning non-linearity as well as whole beam pump depletion. These experiments provide an important test of the predictive capability of the frequency-detuning model, by providing a comparison of saturated ion wave amplitudes and scattering in plasmas in which the ion-wave damping rate is varied from $\mathrm{CH}$ to $\mathrm{Al}$ plasmas. These experiments showed that the saturation of beam amplification with probe beam intensity in a two beam experiment was similar at low intensity in both $\mathrm{Al}$ and $\mathrm{CH}$ plasmas where the ion wave damping rate was different by a factor of $\sim 10$ (i.e., 10\% damping in $\mathrm{CH}$ and $1 \%$ damping in $\mathrm{Al}$ ). The experiments provided evidence that at the highest probe beam intensity (where ion wave amplitudes and scattering are greatest) that the beam amplification was strongly dependent on the ion acoustic wave damping rate, and was in fact completely suppressed in $\mathrm{CH}$ experiments. The non-linear ion wave model explains the persistence of beam amplification in the Al plasma for which the damping rate is low, as shown in Figure 4. Peak values of amplification are determined from the ratio of the Mach +1 and Mach -1 experiments at similar intensities from the waveforms and plotted here with data from experiments with $\mathrm{CH}$ plasmas, and the results of theoretical modeling. Good agreement is obtained for both ion wave damping cases indicating that this newly predicted model can be used as part of a predictive capability for laser-plasma interactions. 


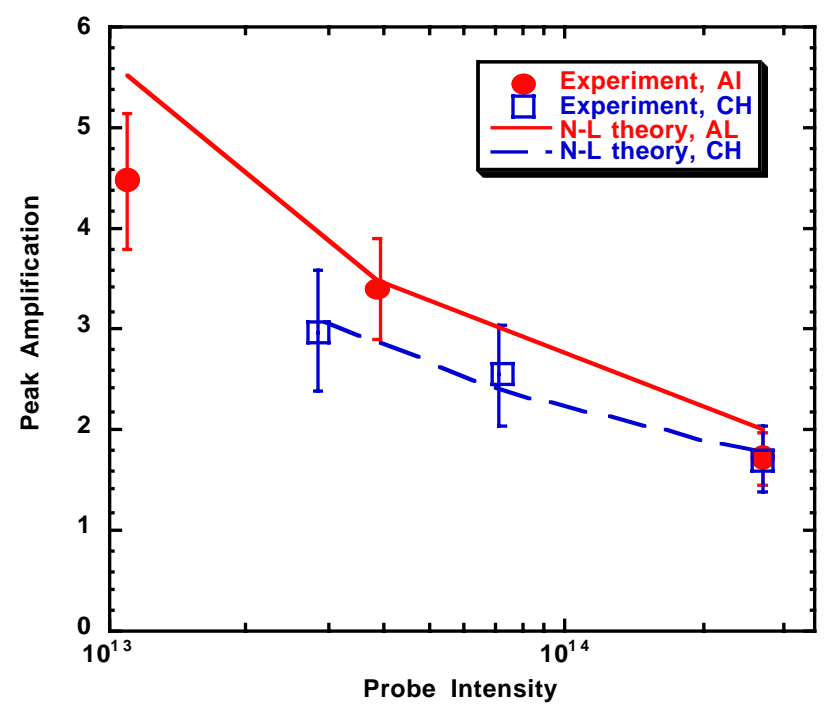

Figure 4. A comparison of the experimentally determined beam amplification (dots) and the predictions of the non-linear ion wave model for $\mathrm{CH}$ and $\mathrm{Al}$ plasmas. The comparison shows persistent $(\sim 2 \mathrm{x})$ beam amplification at the highest probe intensity studied, consistent with saturation of the ion waves by this process.

\section{Experiments on the development of predictive nonlinear saturation models}

\section{a) SBS in low-Z plasmas}

The saturation of ion acoustic waves (for low-Z plasmas) has been investigated with Thomson scattering experiments in two-ion species plasmas consisting of $\mathrm{Be}$ and $\mathrm{Au}$ [3]. In these plasmas, the ion acoustic wave belonging to the Be species has been observed to saturate when driven to large amplitudes by SBS. A novel finding of those studies was the observation of the heating of ions with increasing ion acoustic wave amplitudes. First, this observation was made possible by the use of two-ion species plasmas where accurate measurements of the ion temperature were achieved by Thomson scattering. Second, the heating is a strong indication of ion trapping and possible frequency detuning that has led to the development of our model for low-Z plasmas. Applying the theoretical model shows excellent agreement of the measured ion temperature increase as well as with the experimental SBS reflectivity.

Figure 5 shows the strong increase of the ion to electron temperature ratio as a function of the ion wave amplitude excited by SBS. The shaded area is the result of modeling that infers ion trapping for the saturation of the SBS instability. Also shown is the SBS reflectivity, which decreases with increasing Au concentration as expected from theory. The shaded area represents results of calculations that use the frequency de-tuning model described above and which is based on ion trapping as the dominant saturation mechanism. These calculations give the first quantitative agreement with experimental reflectivity measurements without invoking multipliers. 



Figure 5. Measured and calculated ion temperature increase with increasing ion wave amplitude. This rise is an indication of ion trapping motivating the application of the de-tuning model to calculate the SBS reflectivity. The two different types of data point are obtained with $1 \%$ and $4 \%$ Au concentration. We observe excellent agreement of the de-tuning model with experimental data.

\section{b) SBS in high-Z plasmas}

The saturation of parametric instabilities, such as stimulated Brillouin scattering (SBS) also plays a crucial role for the energy coupling of laser light into large-scale length high$\mathrm{Z}$ plasmas that occur at the hohlraum wall in an ICF experiment. The two-ion wave decay, where a large amplitude ion-acoustic wave decays into two daughter waves, has been postulated as a saturation mechanism for SBS. Measurements of the two-ion decay instability are important to improve our understanding of nonlinear plasma physics. Therefore, in this project, we have performed experiments resulting in the first direct observation of the two-ion decay instability of ion-acoustic waves, driven in a Au plasma by a high intensity laser beam. Using two separate Thomson scattering diagnostics with different scattering geometries simultaneously, we directly measure the thermal ionacoustic fluctuations, the growth of the primary ion-acoustic waves driven by the interaction beam, as well as the secondary two-ion wave decay products.

The experiments were performed at the three-beam Trident laser facility, using a 200 $\mathrm{J} / 1.2 \mathrm{~ns}$ heater beam at $2 \omega$ to create gold plasmas. A second $2 \omega$ interaction beam with an intensity of up to $5 \cdot 10^{15} \mathrm{~W} \cdot \mathrm{cm}^{-2}$ was used to drive ion acoustic waves to large amplitudes, and a $3 \omega$ probe beam was used for Thomson scattering. We employed two separate Thomson scattering diagnostics with different scattering geometries, to match the kvectors of the decay product $\mathbf{k}_{2}$ and the primary driven wave $\mathbf{k}_{\mathrm{ia}}$ simultaneously (Fig. 6a). To fulfill the matching conditions for both Thomson scattering diagnostics and to match the decay product for a large range of decay angles $\alpha$ we used large aperture focusing and collection lenses. We performed k-vector resolved Thomson scattering measurements of 
the decay product, by imaging the collection lens onto the slit of an $0.5 \mathrm{~m}$ imaging spectrometer, coupled to a gated framing camera. For the primary wave diagnostics we imaged target chamber center onto the slit of a second spectrometer, coupled to streak camera.

Figure $1 \mathrm{~b}$ shows the k-vector resolved Thomson scattering two-ion decay measurements. We observe a strong asymmetry between the red and the blue-shifted peak (left), due to collective scattering off ion acoustic waves that are excited to large amplitudes by the two-ion decay intability. This observation is directly correlated with primary ion-acoustic wave amplitudes, driven by SBS. When no primary waves are driven, we observe two symmetric thermal peaks (middle). The separation of the two peaks increases with the average Thomson scattering angle $\Theta$. This increase is well reproduced in the calculated spectrum (right), where we assumed an electron temperature of $950 \mathrm{eV}$.
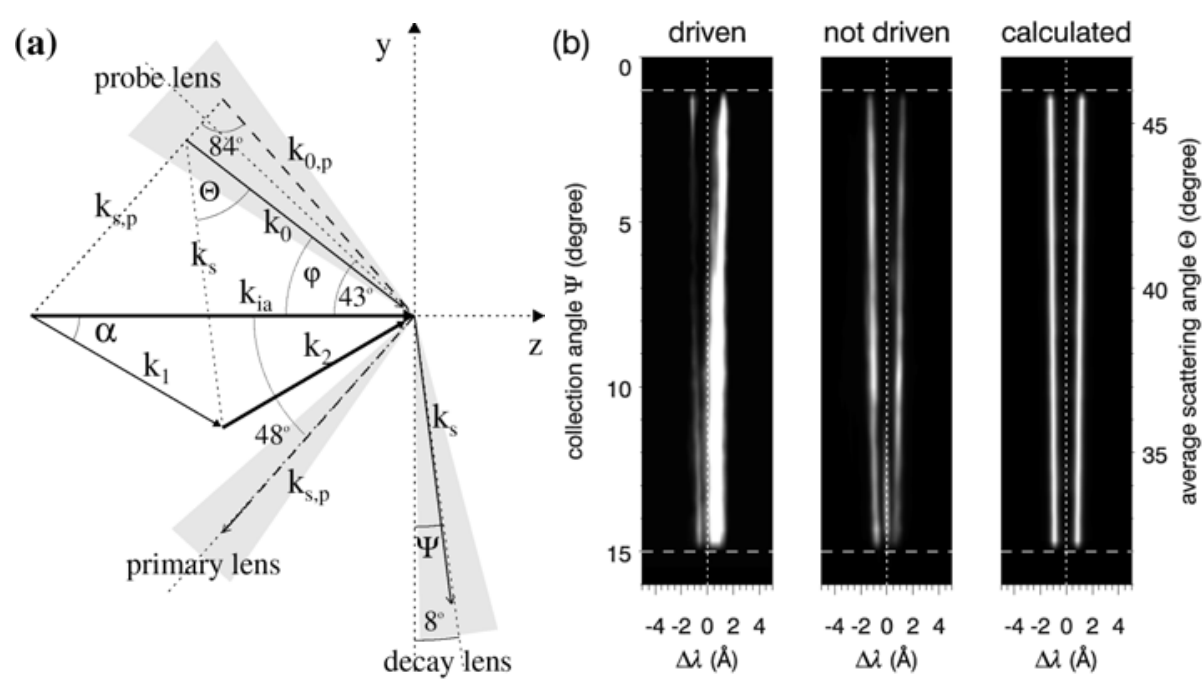

Figure 6. (a) Scattering geometry to match both the primary driven waves $\mathbf{k}_{\mathrm{ia}}$ and the decay product $\mathbf{k}_{2}$ simultaneously. (b) k-vector resolved TS spectra for the case when ion acoustic waves that are driven by the two-ion decay instability to large amplitudes (left), for the case where no waves are driven (middle) and the calculated thermal TS spectrum (right).

In the experiments decay waves are only observed inside the interaction beam volume. We show that secondary decays occur over a broad k-space in accordance with the theory. Two-ion decay processes might thus be energetically important for the saturation of SBS in ICF relevant plasmas. It is clear that a theoretical model that based on two-ion decay and that produces quantitative agreement with the experimental SBS reflectivity in high-Z plasmas (i.e., 5\%) will have to be developed based on these observations. Such an effort is presently underway in the WBS1 project within the ICF program. 


\section{c) SRS in low-Z plasmas}

In this period, we have also succeeded in measuring the properties of the primary plasma waves of the stimulated Raman scattering (SRS) instability with ultraviolet Thomson scattering in large-scale high temperature plasmas. These measurements are critically important to identify the nonlinear effects that determine the SRS reflectivity. Development of Thomson scattering at the OMEGA laser facility has been performed to study the plasma fluid and kinetic response under conditions of interest. Our experiment are now exploring a regime of non-linearity quite distinct from those explored by the LULI and Trident laser facilities primarily because the plasma electron temperature is much higher, the plasma size is much longer, and the plasma is more uniform in density and flow velocity. The sub-kilovolt electron temperatures measured on Trident and LULI have the consequence that electron transport and collisional heating (e.g. inverse bremsstrahlung) are dominant effects in determining the propagation and self-focussing of the laser beams driving the nonlinear effects we are trying to measure. Electron transport in these conditions is poorly understood and, while fundamentally interesting, complicates significantly the analysis of the experimental results. The large scale and uniformity of the Omega laser plasma interaction experiments have provided a medium for instability growth wherein weak growth over an extended volume can be distinguished from strong growth over a short distance by measuring Thomson scattering as well as the reflected and transmitted light. Furthermore, for localized measurements of plasma conditions, active probing with an independent beam is needed.

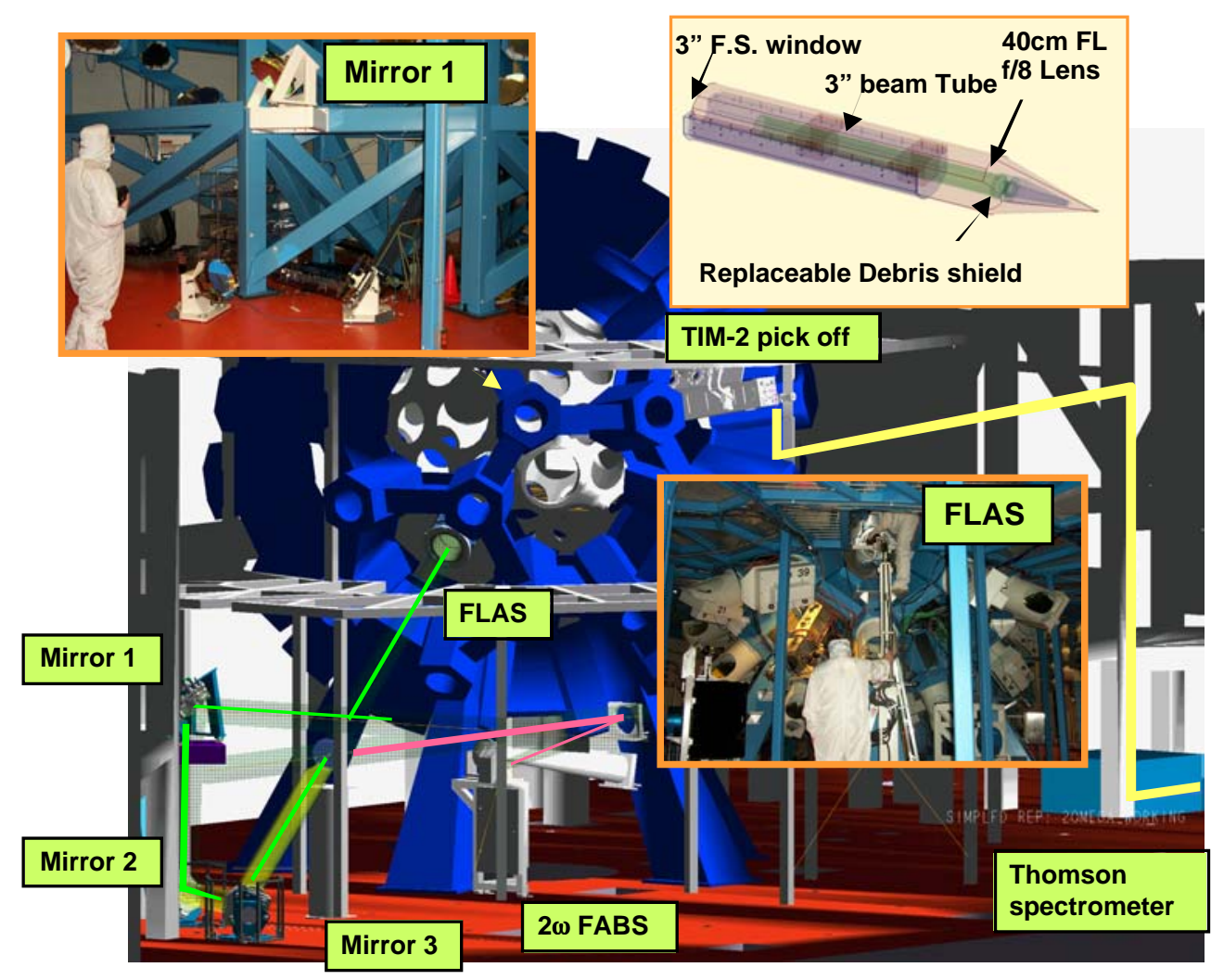

Figure 7. Implementation of the $2 \omega / 4 \omega$ probe laser and Thomson scattering capability at the Omega laser facility, Rochester, NY. 
For these reasons we have implemented a $2 \omega / 4 \omega$ probe laser capability on the Omega laser facility as our essential tool to investigate the nonlinear physics. Figure 7 shows a model drawing of the Omega target chamber and the probe laser. The first insert shows the large transport mirrors that were installed in March 2002. The second insert shows the installation of the final optics assembly (FLAS), which has been demonstrated with a $2 \omega$ as well as a $4 \omega$ lens. The third inset shows the Thomson scattered light pick-off that is inserted into a ten-inch-manipulator to collimate the light so that it can be transported to a streaked spectrometer. The $2 \omega$ capability was successfully activated in June 2002 and the $4 \omega$ capability was successfully activated in July 2003 . We have immediately begun studying saturation in large-scale high temperature gas bag plasmas. In addition, Thomson scattering has also been used as a tool to measure plasma conditions such as the electron temperature.

Data from the activation of the $4 \omega$ probe beam at the Omega Laser Facility are shown in Figure 8. The probe beam operating at a wavelength of $263.5 \mathrm{~nm}$ has been commissioned by using a full aperture KDP quadrupling crystal and a $4 \omega$ focusing lens in the $2 \omega$ probe beam line. The crystal was tuned to convert up to $70 \%$ of the $2 \omega(527 \mathrm{~nm})$ probe beam to $4 \omega(263.5 \mathrm{~nm})$. When operating at full energy the $4 \omega$ probe produces more than $250 \mathrm{~J}$ on target in a $1 \mathrm{~ns}$ pulse. The $4 \omega$ probe was focused, with a new aspheric focusing lens, to a minimum spot size of $50-80 \mu \mathrm{m}$ as shown in Fig. 8 .



Figure 8. X-rays produced by the $4 \omega$ beam focused onto the surface of a $4 \mathrm{~mm}$ diameter gold-coated plastic sphere. The five beams surrounding the probe beam are focused to known locations on the sphere as spatial fiducials (distributed polarization rotators plates give a double spot structure for these beams). 
Thomson scattered light was collected using an f/10 lens and transported via an optical system to the slit of an optical streak spectrograph system. High-quality Thomson scattering data have been measured in a number of collaborative experiments in laser driven hohlraums, planar discs, and gasbag targets (Fig. 9).

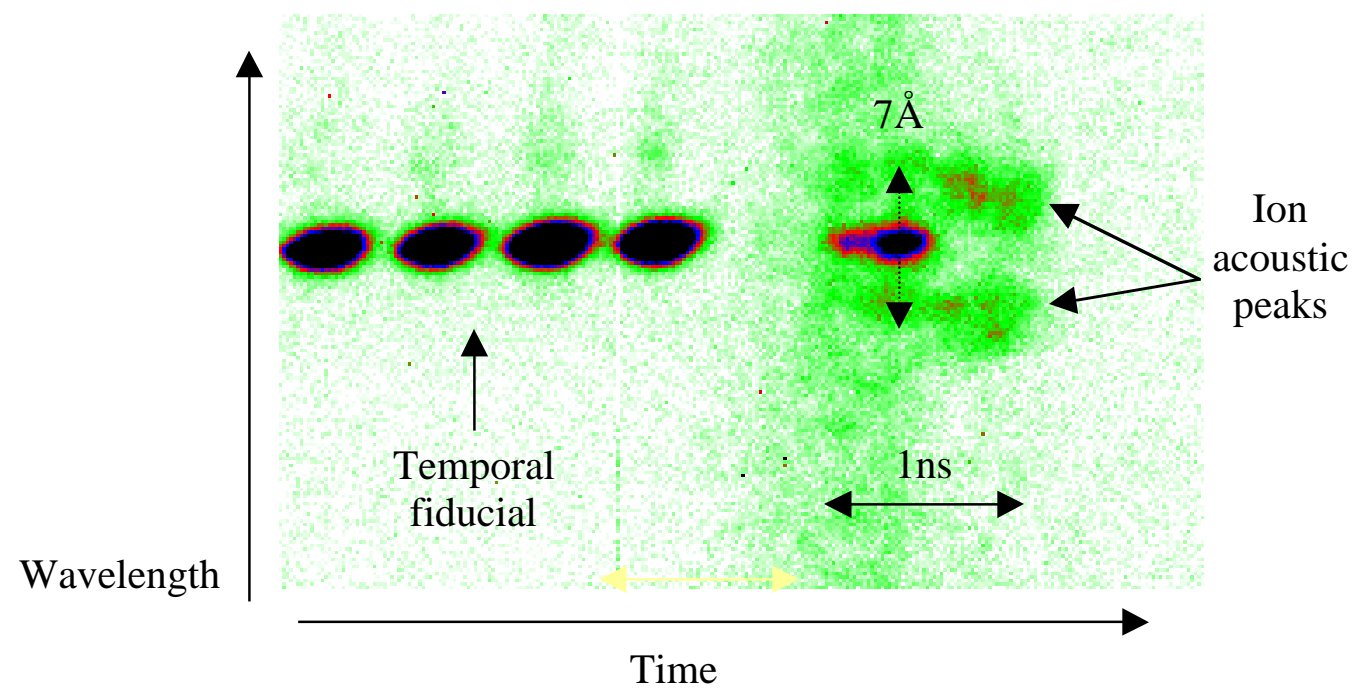

Figure 9. $4 \omega$ Streaked Thomson scattering spectrum from a Krypton gasbag. Preliminary analysis of the spectral shifts of the ion-acoustic features indicates a temperature of $3.5 \mathrm{keV}$.

For example, Fig. 9 shows streaked Thomson scattering spectra from a laser heated gasbag target. Here the two peaks are shifted by the ion-acoustic frequency on either side of the central probe wavelength giving a temperature measurement of $3.5 \mathrm{keV}$.

In this study, we have further performed the first Thomson scattering experiment on electron plasma waves in Omega gasbags (Fig. 10). These measurements have indicated scattering from various electron plasma wave features of the gasbag plasma. The wavelength of the intense feature measured for $0.5 \mathrm{~ns}<\mathrm{t}<1 \mathrm{~ns}$ is consistent with a scattering feature expected from simulations based on the plasma conditions inferred from the SRS scattering.

Figure 10 shows the experimental spectra for experiments where a small amount of $\mathrm{Xe}$ (3\%) was added (right) and those without Xe (left). The scattering signal at wavelengths of 470- $490 \mathrm{~nm}$ is expected from the SRS wavelength shown below in Figure 10. Figure 11 shows the wavelength of the measured Thomson scattering signal peaks and compares them with the expected wavelength shift from the SRS. The agreement is excellent (Fig. 11). This proof-of-principle experiment indicated that the Thomson scattering technique can be used to study the spatial growth of SRS. In the future, the electron plasma wave characteristics will be measured at larger and smaller radii to find out by how much the active SRS region is extended and how it compares with pF3D modeling. Furthermore, experiments measuring the Thomson scattering peaks as function of the SRS reflectivity which is varied by increasing the $2 \omega$ interaction beam intensity, will allow definite tests on the saturation of SRS in high-temperature large scale-length plasmas. 


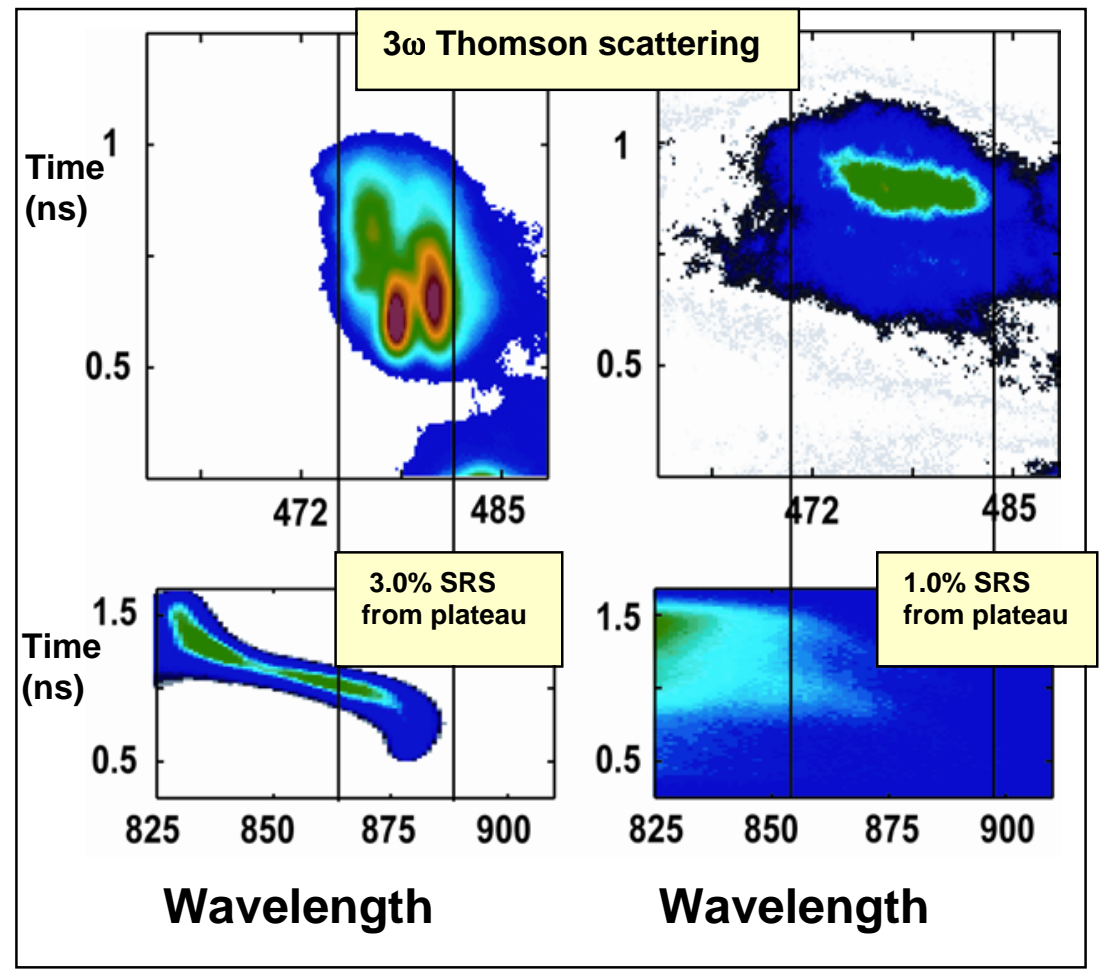

Figure 10. Streak record of Thomson scattered light off the electron plasma wave driven by SRS from the $2 \omega$ interaction beam in a gasbag without Xe (left) and with Xe impurities (right). 


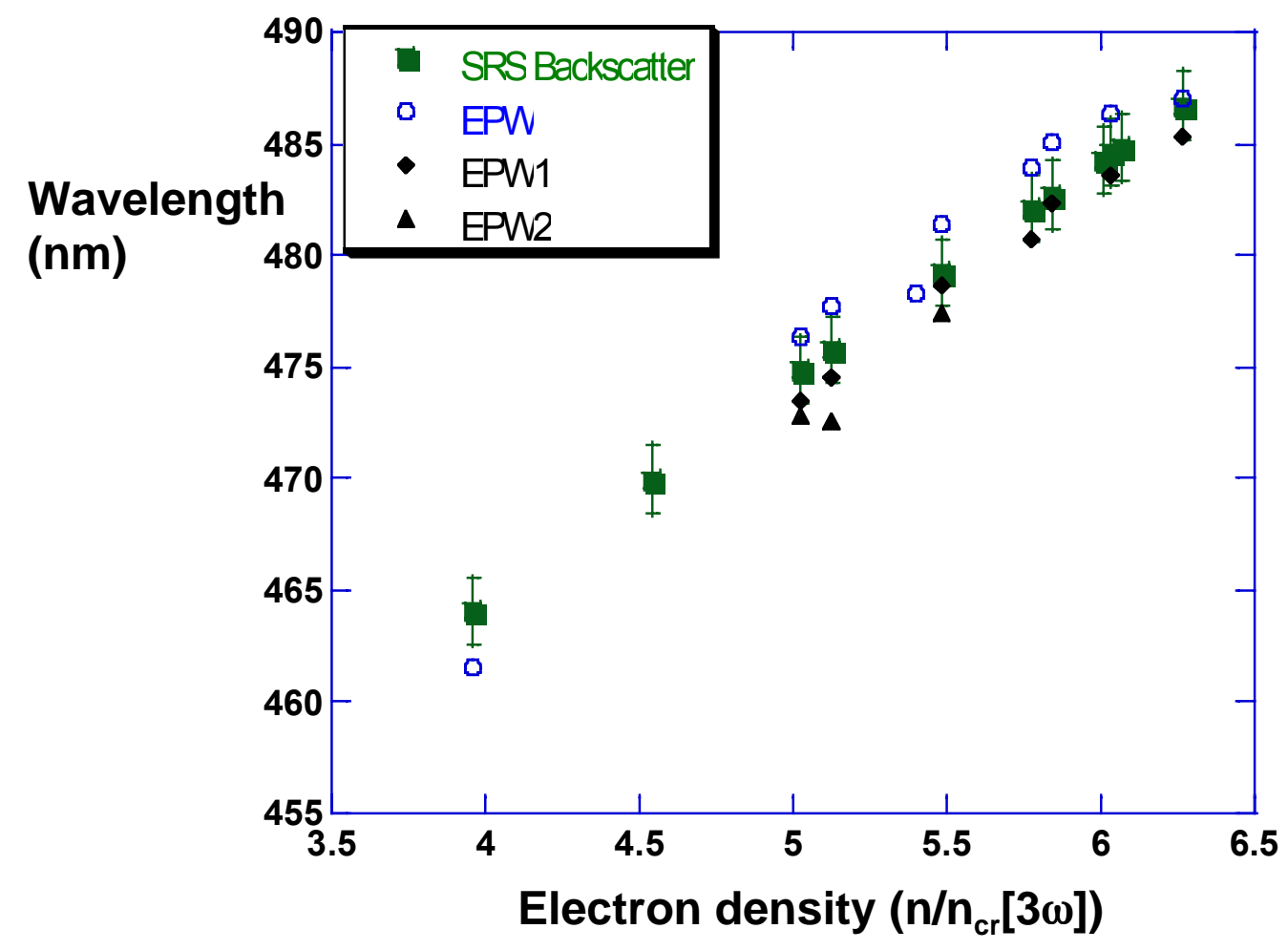

Figure 11. Wavelength of the Thomson scattered light off the electron plasma waves driven by SRS and the expected wavelength shift from SRS. 


\section{References}

[1] S. H. Glenzer, T. L. Weiland, J. Bower, A. J. MacKinnon, and B. J. MacGowan, Rev. Sci. Instrum. 70, 1089 (1999).

[2] S. H. Glenzer, L. M. Divol, R. L. Berger, C. Geddes, R. K. Kirkwood, J. D. Moody, E. A. Williams, and P. E. Young, Phys. Rev. Lett. 86, 2565 (2001).

[3] D. H. Froula et al., to be published (2004).

[4] D. H. Froula, L. Divol, and S. H. Glenzer, Phys. Rev. Lett. 88, 105003 (2002).

[5] D. H. Froula, L. Divol, A. J. MacKinnon, G. Gregori, and S. H. Glenzer, Phys. Rev. Lett. 90, 155003 (2003).

[6] L. Divol, R. L. Berger, B. I. Cohen, E. A. Williams, A. B. Langdon, B. F. Lasinski, D. H. Froula, and S. H. Glenzer, Phys. Plasmas 10, 1822 (2003).

[7] E. A. Williams, B. I. Cohen, L. Divol, M. R. Dorr, J. A. Hittinger, D. E. Hinkel, A. B. Langdon, R. K. Kirkwood, D. H. Froula, and S. H. Glenzer, Physics of Plasmas 11, 231 (2004).

[8] C. Niemann et al., to be pusblished (2004).

[9] R. L. Berger, et al., Physics of Plasmas 5, 4337 (1998).

[10] B. I. Cohen et al., Phys. Plasmas 4, 956 (1997).

[11] S. Brunner, E. Valeo, and J. Krommes, submitted to Phys Rev. Letters (2001); W.L. Kruer, et al., Phys. Rev. Lett. 23, 838 (1969).

[12] C. Labaune, H. A. Baldis, B. S. Bauer, V. T. Tikhonchuk, and G. Laval, Phys. Plasmas 5, 234 (1998).

[13] D. S. Montgomery, R. P. Johnson, H. A. Rose, J. A. Cobble, and J. C. Fernandez, Phys. Rev. Lett. 84, 678 (2000).

[14] R. K. Kirkwood, J. D. Moody, A. B. Langdon, B. I. Cohen, E. A. Williams, M. R. Dorr, J. A. Hittinger, R. L. Berger, P. E. Young, L. J. Suter, L. Divol, S. H. Glenzer, O. L. Landen, and W. Seka, Phys. Rev. Lett. 89, 215003 (2002). 


\section{Appendix: Published papers and talks in 01-ERD-107}

\begin{tabular}{|c|c|c|c|c|}
\hline Glenzer & UCRL-CONF-155301 & Progress in long scale length laser-plasma interactions & $\begin{array}{l}\text { 3rd International Conference on } \\
\text { Inertial Fusion Sciences and } \\
\text { Applications (IFSA 2003), } \\
\text { Monterey, CA 9/7-12/03 }\end{array}$ & $\begin{array}{l}\text { Conference } \\
\text { document }\end{array}$ \\
\hline & UCRL-JC-135822-REV2 & & & \\
\hline & UCRL-JC-144618-ABS & & & \\
\hline Froula & UCRL-JC-145885 & $\begin{array}{l}\text { Observation of ion heating by stimulated brillouin scattered } \\
\text { driven ion-accoustic waves using Thomson scattering }\end{array}$ & Physics of Plasmas & Journal doc \\
\hline Divol & UCRL-JC-148447-ABS & $\begin{array}{l}\text { Nonlinear behavior of Stimulated Brillouin Scattering in 1D-PIC } \\
\text { simulations of a } 500(\mathrm{Mu}) \mathrm{m} \text { long Be plasma }\end{array}$ & $\begin{array}{l}\text { 32nd Anomalous Absorption } \\
\text { Conference 2002, Oahu, HI 7/21- } \\
26 / 02\end{array}$ & Abstract \\
\hline Froula & UCRL-JC-148448-ABS & $\begin{array}{l}\text { Search for SBS saturation processes with Thomson scattering } \\
\text { experiments on ion-acoustic waves in Beryllium plasmas }\end{array}$ & $\begin{array}{l}\text { 32nd Anomalous Absorption } \\
\text { Conference 2002, Oahu, HI 7/21- } \\
26 / 02\end{array}$ & Abstract \\
\hline Froula & UCRL-JC-148448-ABS & $\begin{array}{l}\text { Search for SBS saturation processes with Thomson scattering } \\
\text { experiments on ion-acoustic waves in Beryllium plasmas }\end{array}$ & $\begin{array}{l}\text { 32nd Anomalous Absorption } \\
\text { Conference 2002, Oahu, HI 7/21- } \\
26 / 02\end{array}$ & Abstract \\
\hline Divol & UCRL-JC-148980-ABS & $\begin{array}{l}\text { Detailed pictures of the saturation of SBS driven acoustic waves } \\
\text { in laser heated plasmas }\end{array}$ & $\begin{array}{l}\text { 44th Annual Meeting of the Division } \\
\text { of Plasma Physics, Orlando, FL } \\
11 / 11-15 / 02\end{array}$ & Abstract \\
\hline $\begin{array}{l}\text { Divol/ } \\
\text { Mounaix }\end{array}$ & UCRL-JC-148982 & $\begin{array}{l}\text { Near threshold reflectivity fluctuations in the independent- } \\
\text { convective-hot-spot limit of a spatially smoothed laser beam }\end{array}$ & Physical Review Letters & Journal doc \\
\hline Divol & UCRL-JC-148983-SUM & $\begin{array}{l}\text { Workshop on the Nonlinear Saturation of Stimulated Raman and } \\
\text { Brillouin Instabilities for Plasma and Laser Parameters of Interest } \\
\text { of Inertial Fusion Confinement and High Density Science }\end{array}$ & $\begin{array}{l}\text { Workshop on the Nonlinear } \\
\text { Saturation of SBs/SRS, Wente } \\
\text { Vineyards, } 4 / 3-5 / 02\end{array}$ & Summary \\
\hline Froula & UCRL-JC-149002-ABS & $\begin{array}{l}\text { Laser-plasma interaction experiments in the nonlinear saturated } \\
\text { regime }\end{array}$ & $\begin{array}{l}\text { 44th Annual Meeting of the Division } \\
\text { of Plasma Physics, Orlando, FL } \\
11 / 11-15 / 02\end{array}$ & Abstract \\
\hline Divol & UCRL-JC-149983 & $\begin{array}{l}\text { Nonlinear saturation of Stimulated Brillouin Scattering for long } \\
\text { time scales }\end{array}$ & Physics of Plasmas & Journal doc \\
\hline Froula & UCRL-JC-150767 & Direct observation of SBS detuning by a velocity gradient & Physical Review Letters & Journal doc \\
\hline
\end{tabular}




\begin{tabular}{|c|c|c|c|c|}
\hline Glenzer & UCRL-JC-151526-ABS & $\begin{array}{l}\text { Results from simultaneous } 527 \mathrm{~nm} \text { and } 351 \mathrm{~nm} \text { probe beam } \\
\text { interactions in a long scale-length plasmas }\end{array}$ & $\begin{array}{l}\text { 5th International Workshop on } \\
\text { Laser-Plasma Interaction Physics, } \\
\text { Banff, Canada, } 2 / 19-22 / 03\end{array}$ & Abstract \\
\hline Froula & UCRL-JC-151527-ABS & Stimulated Brillouin scattering from Helium-Hydrogen plasmas & $\begin{array}{l}\text { 5th International Workshop on } \\
\text { Laser-Plasma Interaction Physics, } \\
\text { Banff, Canada, } 2 / 19-22 / 03\end{array}$ & Abstract \\
\hline Divol & UCRL-JC-151529-ABS & $\begin{array}{l}\text { A reduced model of kinetic effects related to the saturation of } \\
\text { stimulated brillouin scattering in long plasmas }\end{array}$ & $\begin{array}{l}\text { 5th International Workshop on } \\
\text { Laser-Plasma Interaction Physics, } \\
\text { Banff, Canada, 2/19-22/03 }\end{array}$ & Abstract \\
\hline Divol & UCRL-JC-151529-ABS & $\begin{array}{l}\text { A reduced model of kinetic effects related to the saturation of } \\
\text { stimulated brillouin scattering }\end{array}$ & $\begin{array}{l}\text { IFSA - Previously bluesheeted for } \\
\text { other purpose }\end{array}$ & Abstract \\
\hline Divol & UCRL-JC-154237-ABS & $\begin{array}{l}\text { Detailed analysis of backscattering measuremennts for } 351 \mathrm{~nm} \\
\text { light from gasbag targets }\end{array}$ & $\begin{array}{l}\text { 45th Annual Meeting of the Division } \\
\text { of Plasma Physics, Albuquerque, NM } \\
10 / 27-31 / 03\end{array}$ & Abstract \\
\hline \multirow[t]{6}{*}{ Divol } & UCRL-JC-155169 & \multicolumn{2}{|c|}{ A reduced model of kinetic effects related to the saturation of stimulated brillouin scattering } & \\
\hline & \multicolumn{2}{|l|}{ UCRL-PRES-143543-REV1 } & & \\
\hline & UCRL-PRES-144618 & & & \\
\hline & \multicolumn{2}{|l|}{ UCRL-PRES-145885-REV1 } & & \\
\hline & UCRL-PRES-146488 & & & \\
\hline & UCRL-PRES-147839 & & & \\
\hline \multirow[t]{2}{*}{ Glenzer } & UCRL-PRES-147840 & Cocktail hohlraum experiments & $\begin{array}{l}\text { Workshop on the Nonlinear } \\
\text { Saturation of SBs/SRS, Wente } \\
\text { Vineyards, } 4 / 3-5 / 02\end{array}$ & $\begin{array}{l}\text { Viewgraph } \\
\text { s }\end{array}$ \\
\hline & UCRL-PRES-147841 & & & \\
\hline Divol & UCRL-PRES-148447 & $\begin{array}{l}\text { Nonlinear behavior of Stimulated Brillouin Scattering in 1D-Hybrid } \\
\text { PIC simulations of a } 500(\mathrm{Mu}) \mathrm{m} \text { long Be plasma }\end{array}$ & $\begin{array}{l}\text { 32nd Anomalous Absorption } \\
\text { Conference 2002, Oahu, HI 7/21- } \\
26 / 02\end{array}$ & $\begin{array}{l}\text { Viewgraph } \\
\text { s }\end{array}$ \\
\hline \multirow[t]{2}{*}{ Divol } & UCRL-PRES-148978 & $\begin{array}{l}\text { Nonlinear behavior of Stimulated Brillouin Scattering: Experiments, } \\
\text { detailed simulations and reduced models }\end{array}$ & $\begin{array}{l}\text { LLNL HEDES Summer School, Santa } \\
\text { Cruz, CA }\end{array}$ & $\begin{array}{l}\text { Viewgraph } \\
\text { S }\end{array}$ \\
\hline & UCRL-PRES-148978 & & & \\
\hline Divol & UCRL-PRES-148980 & \multicolumn{2}{|c|}{ Detailed pictures of the saturation of SBS driven acoustic waves in laser heated plasmas } & $\begin{array}{l}\text { Viewgraph } \\
\text { S }\end{array}$ \\
\hline Divol & UCRL-PRES-153098 & \multicolumn{2}{|c|}{$\begin{array}{l}\text { Kinetic effects related to the saturation of stimulated brillouin backscatteirng (SBS) and their modeling } \\
\text { in the fluid code } \mathrm{pF} 3 \mathrm{~d}\end{array}$} & $\begin{array}{l}\text { Viewgraph } \\
\text { S }\end{array}$ \\
\hline
\end{tabular}




\begin{tabular}{|c|c|c|c|c|}
\hline Divol & $\begin{array}{l}\text { UCRL-PRES-153098- } \\
\text { REV1 }\end{array}$ & $\begin{array}{l}\text { Kinetic effects related to the saturation of stimulated brillouin } \\
\text { backscatteirng (SBS) and their modeling in the fluid code pF3d }\end{array}$ & $\begin{array}{l}\text { International Conference on } \\
\text { Strongly Coupled Coulomb Systems, } \\
\text { Sante Fe, NM 9/2-6/02 }\end{array}$ & $\begin{array}{l}\text { Viewgraph } \\
\text { s }\end{array}$ \\
\hline Divol & $\begin{array}{l}\text { UCRL-PRES-153098- } \\
\text { REV2 }\end{array}$ & $\begin{array}{l}\text { Kinetic effects related to the saturation of stimulated brillouin } \\
\text { backscatteirng (SBS) and their modeling in the fluid code pF3d }\end{array}$ & $\begin{array}{l}\text { 3rd International Conference on } \\
\text { Inertial Fusion Sciences and } \\
\text { Applications (IFSA 2003), } \\
\text { Monterey, CA 9/7-12/03 } \\
\end{array}$ & $\begin{array}{l}\text { Viewgraph } \\
\text { s }\end{array}$ \\
\hline Glenzer & UCRL-PRES-153100 & \multicolumn{2}{|c|}{$\begin{array}{l}\text { In collaboration with LLE, we have implemented a } 2 w / 4 w \text { Thomson scattering capability at the Omega } \\
\text { facility }\end{array}$} & $\begin{array}{l}\text { Viewgraph } \\
\text { s }\end{array}$ \\
\hline
\end{tabular}

